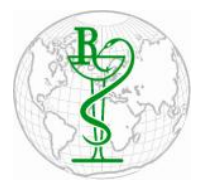

INDO GLOBAL JOURNAL OF

PHARMACEUTICAL SCIENCES

ISSN 2249- 1023

\title{
Pathophysiology of Parkinson: An Updated Review
}

\author{
Sandeep Kumar* \\ Department of Pharmaceutical Sciences, Maharshi Dayanand University, Rohtak, Haryana, India
}

\begin{abstract}
Address for Correspondance Sandeep Kumar, sranga118@g mail.com
\end{abstract}

\section{Received:}

22.02.2018

Accepted:

18.03.2018

\section{Keywords}

Dopamine;

Neuromelanin;

Nitric Oxide;

Parkinson.

\begin{abstract}
Nitric oxide (NO) is an endogenous molecule which functions as a neurotransmitter, hormone, free radical, etc. NO has been found to regulate the release of neurotransmitters, synaptic transmission, cell death, etc. NO is involved in the pathogenesis of various neuropsychiatric and neurodegenerative disorders. NO plays a key role in cellular apoptosis and neuronal degeneration. Parkinson' disease (PD) is a neurodegenerative disorder characterized by motor dysfunction that can be seen in the patients suffering from PD. The motor dysfunction is due to the progressive degeneration of dopaminergic neurons in mid brain. Dopamine (DA) is highly reactive molecule and is prone to the oxidation very much. The oxidation of DA is accompanied by the production of the reactive oxygen species that activates microglia cells. Upon activation, microglia cells cause the upregulation of inducible NO synthase, the enzyme involved in the production of NO. NO thus plays a key role in the neurodegeneration process implicated in PD. Thus, the aim of the present manuscript is to describe the possible role of NO in PD. () 2018 iGlobal Research and Publishing Foundation. All rights reserved.
\end{abstract}

Cite this article as: Kumar, S. Pathophysiology of Parkinson: An Updated Review. Indo Global J. Pharm. Sci., 2018; 8(3): 108-114.

\section{INVOLVEMENT OF NO IN THE PATHOGENESIS OF PD}

Excessive NO synthesis and parkinsonism authenticate the statistical implications of NOS1 and NOS2A in PD [1]. Also there is a significant increase in the CSF nitrite content in the PD patients. Raised levels of 3-NT formed by the NO attack on free tyrosine and tyrosine has been found in proteins in PD [2]. Pharmacological inhibition of iNOS has been shown to prevent dopaminergic neurodegeneration as a consequence of microglial activation and transgenic mice lacking iNOS are more resistant to MPTP-mediated dopaminergic neurotoxicity [3]. Activated microglia secretes various pro-inflammatory mediators including cytokines such as IL- 6 and TNF- $\alpha$, reactive oxygen species, and reactive nitrative species such as NO. These factors are believed to contribute to microgliamediated neurotoxicity [3]. Selective inhibition of NF- $\mathrm{kB}$ activation suppressed microglial activation and prevented DA neuronal loss against MPTP-injured PD mouse model. Fluoxetine significantly inhibited LPS-induced NF- $\mathrm{BB}$ activation, which might be responsible for the decreased production of pro-inflammatory factors and consequent neuroprotection [4]. A common feature observed in the neuronal cells of $\mathrm{AD}$ and $\mathrm{PD}$ victims in this sporadic variant was the attachment of nitric oxide (NO) to the redox-active cysteines of protein-disulfide isomerase (PDI) to form SNitroso PDI. The formation of S-nitroso-PDI coupled with the making the oxidoreductase a chief target for the prevention of these two neurodegenerative disorders in the nitrosativestress-linked variant of the diseases. PDI is severely compromised by the addition of nitric oxide resulting from elevated levels of nitrosative stress [5]. 


\section{Indo Global Journal of Pharmaceutical Sciences, 2018; 8(3): 108-114}

Brain appears to be particularly susceptible to oxidative stress due to its heavy oxygen demands but the nigra seems to be highly susceptible to oxidative stress presumably due to its large population of dopaminergic neurons, which produce abundant quantities of ROS. There is a necessary fine-tuned balance between the extent of the production of and the removal of oxidants, and it is this balance that keep ROS and RNS constantly at low, non-toxic levels. Thus PD is associated with a higher production of or a lower detoxification of oxidants [6]. The presence of oxidative stress in PD has been associated with increased oxidation of lipids, DNA and proteins and the generation of ROS. Furthermore, oxidative stress has been demonstrated in PD sufferers and evidence also clearly supports the involvement of impaired mitochondrial function in PD [7]. The total of $1 \%$ of all oxygen consumption might be reduced to superoxide or hydrogen peroxide. However, neither superoxide nor hydrogen peroxide is particularly toxic. Cells can greatly increase the toxicity of superoxide by producing nitric oxide [7].

The death of the dopaminergic neurons in the $\mathrm{SN}$ results in the accumulation of extracellular NM and NM activate microglia, suggesting a proinflammatory role for NM. NM activates microglia by triggering NK- $\mathrm{kB}$ activation [8]. Upon activation, microglia proliferate, undergo morphogenesis, and increase cell volume with extension of their processes. Activated microglia are well adapted for the induction of inflammation, cytokine-mediated and antibody-dependent cell cytotoxicity. These toxic substances include reactive oxygen species (ROS), reactive nitrogen species (RNS), proinflammatory cytokines, and prostaglandins. Uncontrolled microglial activation is toxic to neurons, due in part to release of pro-inflammatory factors including, but not limited to interleukin one beta (IL-1 $\beta$ ), tumor necrosis factor alpha (TNF- $\alpha$ ), IL-6, nitric oxide (NO), prostaglandin E2 (PGE2), and superoxide radical [9].

TNF-Alpha, a pro-inflammatory cytokine, is produced by many cell types, including macrophages, lymphocytes, fibroblasts and keratinocytes, in response to inflammation, infection, and other environmental stresses. TNF-Alpha acts by binding to its receptors, TNFR1 (p55) and TNFR2 (p75), on the cell surface. Most cells express TNFR1, which is believed to be the major mediator of the cytotoxicity of TNFAlpha $[10,11]$. Binding of TNF-Alpha to its two receptors, TNFR1 and TNFR2, results in recruitment of signal transducers that activate at least three distinct effectors. Through complex signaling cascades and networks, these effectors lead to the activation of Caspases and two transcription factors, Activation Protein-1 and NF-KappaB
(Nuclear Factor-KappaB). Initially, TRADD (TNFRAssociated Death Domain) protein, binds to TNFR1, then, TRADD recruits FADD (Fas-Associated Death Domain). Binding of TRADD and FADD to TNFR1 leads to the recruitment, oligomerization, and activation of Caspase8. Activated Caspase8 subsequently initiates a proteolytic cascade that includes other Caspases (Caspases3,6,7) and ultimately induces apoptosis. Caspase8 also cleaves BID (BH3 Interacting Death Domain). tBID (Truncated BID) disrupts the outer mitochondrial membrane to cause release of the proapoptotic factors CytoC (Cytochrome-C). CytoC that is released from the intermembrane space binds to APAF1 (Apoptotic Protease Activating Factor-1), which recruits Caspase9 and in turn can proteolytically activate Caspase3. A novel $61-\mathrm{kDa}$ protein kinase RIP2 is related to RIP that is a component of TNFR1 which mediates the recruitment of Caspase death proteases. TRAF2 (TNF Receptor-Associated Factor-2) has been implicated in the activation of two distinct pathways that leads to the activation of Activation Protein-1 via the JNK (Jun NH2-terminal Kinase), MEKK (MEK Kinase), p38 and, together with RIP, NF-KappaB activation, via the NIK (NF-KappaB-Inducing Kinase). Overexpression of SODD (Silencer Of Death Domains), a $60 \mathrm{kDa}$ protein, associated with the DD of TNFRI suppresses TNF-induced cell death and NF-KappaB activation demonstrating its role as a negative regulatory protein for these signaling pathways [12]. Expression of NOS2 in diverse cell types is highly dependent on the NF- $\kappa B$ signaling pathway and we demonstrated previously a requirement for $\mathrm{NF}-\mathrm{\kappa B}$ in the expression of NOS2 in activated astrocytes after stimulation with inflammatory cytokines and manganese. TNF- $\alpha$ induced activation of NFkB and thus is responsible for NFKB mediated iNOS induction [13].

Astrogliosis results in increased production of various neurotoxic inflammatory mediators, including nitric oxide (NO) that contributes to progressive loss of nigrostriatal neurons. Supporting a deleterious role for excessive NO production in PD are postmortem observations of increased NOS2 expression in patients diagnosed with PD, as well as reports that deletion of the Nos2 gene in mice confers protection against MPTP-mediated neurotoxicity. Microglial cells expresses high levels of iNOS in the SN of PD patients compared with control patients. iNOS is known to mediate NO production, which causes neuronal toxicity. Thus, it is suggested that toxic NO levels may occur in close proximity to dopaminergic neurons [14]. The important target of mitochondrial superoxide is NO. The spontaneous reaction of superoxide with $\mathrm{NO}$ occurs at a rate comparable to that with SOD2 and generates peroxynitrite, a powerful oxidizing species. Cellular damage induced by peroxynitrite includes 


\section{Indo Global Journal of Pharmaceutical Sciences, 2018; 8(3): 108-114}

depletion of thiol-dependent antioxidants, DNA strand breakage, lipid oxidation, and protein nitration [15].

Prx family proteins are important antioxidant enzymes that limit accumulation of intracellular peroxides. Active Prx2 reduces $\mathrm{H}_{2} \mathrm{O}_{2}$ and other peroxides by using normal redox cycle and detoxifies intracellular peroxides. Prx2 activity is regulated by NO. S-nitrosylation targets the redox-active Cys51 and Cys-172 residues and forms SNO-Prx2 which results in the generation of nonfunctional Prx2, which furthers results in accumulation of cellular peroxides that further oxidize SNO$\operatorname{Prx} 2$ to $\operatorname{Prx} 2-\mathrm{SO}_{2 / 3} \mathrm{H}$. Interfering with the normal antioxidant system by the generation of SNO-Prx2 may contribute to neuronal cell death. NO-mediated pathway leading to neuronal cell death involving Prx2 dysfunction may lead to the development of new therapeutic approaches for sporadic PD and other neurodegenerative disorders associated with nitrosative stress [16]. XIAP is an antiapoptotic protein that is known to be crucial for cell survival. The mechanism by which S-nitrosylation of parkin impairs its protective function appears to occur through inhibition of its E3 ubiquitin ligase activity. This idea contrasts with the mechanism of impairment of XIAP antiapoptotic function by S-nitrosylation. XIAP antiapoptotic function is inhibited by S-nitrosylation through preventing XIAP's binding to caspase-3. Neuroprotective proteins such as peroxiredoxin and proteindisulphide isomerase are modified by S-nitrosylation, and this modification compromises their normal protective functions. Thus nitrosative stress is an important contributor in the pathogenesis of PD [17].

Lewy bodies are the neuropathological hallmark of the diagnosis and pathophysiology of the neurodegenerative process [18]. $\alpha$-Syn is the main protein component of Lewy bodies, but, importantly, the $\alpha$-syn found in Lewy bodies is misfolded and phosphorylated, indicating that a pathogenic variety of $\alpha$-syn accumulates in diseased or damaged cells. The native $\alpha$-syn monomer is an unfolded, soluble protein, and in vitro studies have shown that monomeric, oligomeric, and fibrillar species of $\alpha$-syn exist in equilibrium [19]. Oligomerization of asyn initiates with the dimerization of partially folded monomers, followed by the formation of $\beta$ sheet rich nonfibrillar, oligomeric intermediates, also known as protofibrils. Protofibrils are transient $\beta$-sheet-containing oligomers that are formed during aggregation. Studies have shown that the presence of the disease-associated mutations in asyn increase rates of self-assembly and fibrillization [19, 20]. Its aggregation is involved in Lewy body formation and subsequent neuronal cell death. Oxidized glutathione (GSSG) is known to accelerate this aggregation and neuronal death [21]. The importance of extracellular aSyn is further supported by the role of aSyn in triggering neuroinflammatory glial responses. Extracellular aggregated aSyn added to neuron-glia culture lead to microglia activation and enhanced dopaminergic neurodegeneration [22]. $\alpha$-synuclein act as a negative regulator of dopamine release, perhaps by modulating vesicle fusion and distribution. Thus the overexpression of $\alpha$-synuclein decrease the rate of dopamine release [23]. Nitrated $\alpha$-synuclein has also been detected in the SN and ventral midbrain of mice treated with MPTP Recombinant $\alpha$-synuclein treated with nitrating agents (peroxynitrite $/ \mathrm{CO}_{2}$ myeloperoxidase $/ \mathrm{H}_{2} \mathrm{O}_{2} /$ nitrite) generates highly stable nitrated $\alpha$-synuclein oligomers as a consequence of oxidation resulting in the formation of crosslinked o-o'ditryrosine. This form of aggregated $\alpha$-synuclein is resistant to proteolysis, perhaps due to its structural stabilization. Nitrated $\alpha$-synuclein activated $\mathrm{T}$ cells enhances microglial activation and degeneration of dopaminergic neurons. This suggests that oxidative/nitrative PTMs may cause an adaptive immune response that augments the neuropathology in PD [24].

\section{NO MEDIATED NEURODEGENERATION IN PD}

NO plays key role in neurotransmission but at higher concentrations, it is implicated in the pathogenesis of stroke, demyelination, and other neurodegenerative diseases. NO derived from activated glial cells is assumed to contribute to oligodendrocyte degeneration in demyelinating diseases and neuronal death during ischemia, trauma and neurodegenerative diseases [25]. Microglia are the major resident immune cells in the brain and under physiological conditions, microglia exhibit a deactivated phenotype that is associated with the production of anti-inflammatory and neurotrophic factors. Microglia switch to an activated phenotype in response to pathogen invasion or tissue damage and thereby promote an inflammatory response that serves to further engage the immune system and initiate tissue repair [26]. Mechanisms by which activated microglia kill neurons include the increased expression of iNOS in glia [27].

NOS induction is predominantly regulated by the two proinflammatory transcription factors NF-кB [28]. Singlenucleotide genetic polymorphisms in the TNF promoter that increase transcriptional activity and TNF production are associated with earlier onset of disease in patients with idiopathic PD. Also TNF mRNA and protein levels are elevated in postmortem brain and cerebrospinal fluid. Soluble TNF (solTNF) is the ligand species mediating the cytotoxic effects on DA neurons [29]. TNF- $\alpha$, is rapidly produced in the brain in response to tissue injury. TNF- $\alpha$ induced activation of 


\section{Indo Global Journal of Pharmaceutical Sciences, 2018; 8(3): 108-114}

$\mathrm{NF}-\mathrm{kB}$ and thus is responsible for NF- $\mathrm{kB}$ mediated iNOS induction [13]. CD23-dependent activation of iNOS in microglial cells may be involved in the cascade of events leading to DA cell death. However, the principal ligand for CD23-IgE has not been detected in the SN of PD patients and it is thought that other likely ligands such as the $\alpha$ chain CD11b and CD11c of the adhesion molecules CD11b/CD18 and CD11c/CD18 may trigger CD23 activation in PD [14].

Increased expression of iNOS results in the increased production of NO. It is believed that NO can react with superoxide radicals forming the highly toxic peroxynitrite, which causes nitration of tyrosine residues (3-nitrotyrosine) on cellular proteins resulting in both structural and functional alterations. PN can also cause protein damage by modifying tyrosine (3-nitrotyrosine formation, 3NT), cysteine ( $S$ nitrosylation or SNO formation), or tryptophan (via formation of $N$-formylkynurenine) residues. The significance of this neurotoxic mechanism is supported by studies that demonstrate increased 3-nitrotyrosine immunostaining in Lewy bodies in PD patients and also the presence of increased 3-nitrotyrosine in MPTP-lesioned mice [14, 30]. Levels of 3nitrotyrosine, a biomarker of attack by $\mathrm{ONOO}^{-}$and other reactive nitrogen species upon protein-bound tyrosine residues, were also significantly increased in the mutant Parkin transfectants, again independent of Parkin enzyme activity. The mutant proteins could elevate levels of $\mathrm{NO} / \mathrm{NO}_{3}^{-}$, for which increased expression of nNOS (but not iNOS) was presumably responsible.

Proteasomal dysfunction may be a significant contributor to neuronal cell death in the major neurodegenerative diseases [31]. NO is known to inhibit several enzymes including complexes I and IV of the mitochondrial electron transport chain and aconitase [30]. NO can exacerbate neuronal injury resulting from excitotoxicity. Increased intracellular $\mathrm{Ca}^{2+}$ levels, resulting from activation of glutamate receptors such as NMDA receptors, stimulate nNOS to produce more NO. The continual influx of $\mathrm{Ca}^{2+}$ through the open NMDA receptors compounds the stress on mitochondria, which attempt to sequester and buffer $\mathrm{Ca}^{2+}$. As mitochondrial membrane potential decreases because of the overflow of $\mathrm{Ca}^{2+}$, mitochondria reverse their ATP synthase in an attempt to restore it. Eventually, the excess $\mathrm{Ca}^{2+}$ uptake causes mitochondrial membrane potential loss, mitochondrial swelling, opening of the mitochondrial permeability transition pore, outer membrane rupture, and spill of $\mathrm{Ca}^{2+}$ and apoptogenic factors into the cytoplasm [32]. $\mathrm{Ca}^{2+}$ loads in the matrix sensitize the mPTP to apoptotic stimuli [33].
Mitochondria have been identified as critical for the apoptotic process. Mitochondrion integrates death signals engaged by proteins in the $\mathrm{Bcl}-2$ family and releases pro-apoptotic molecules residing in the mitochondrial intermembrane space to activate caspases leading to internucleosomal cleavage of DNA. Apoptotic stimuli produce conformational changes and oligomerization of pro-apoptotic proteins (Bax, Bak, etc.) and destabilizes lipid bilayer, creating pores which results into the release of cytochrome $c$ into the cytoplasm, which induces the formation of the Apaf1-containing macromolecular complex called the apoptosome. Released cytochrome $c$ then triggers the assembly of the cytoplasmic apoptosome a protein complex of apoptotic protease activating factor 1 (Apaf1), cytochrome $c$, and procaspase-9. This is the engine that drives caspase-3 activation. [34]. Caspases contain a single cysteine at the catalytic site, which is susceptible to redox modification and can be effectively modified by $S$-nitrosylation in the presence of NO. $S$-nitrosylated caspases can be a source to transfer of NO to XIAP via transnitrosylation and contribute to an additional mechanism to produce SNO-XIAP, inhibit its E3 ubiquitin ligase activity, leading to neuronal death. Also the Caspase activation during NO stimulation also occurs as a result of down-regulation of X-linked inhibitor of apoptosis protein (XIAP) which interacts with active caspases-3/-7/-9 in the cytosol and inhibit the catalytic activity of apoptotic caspases [35].

\section{NO MEDIATED NEURONAL PROTECTION IN PD}

An unbalanced generation of RNS as a feature of PD pathology. Glial cells within the $S N$ exhibit increased NO levels, possibly due to the accumulation of interferon- $\gamma$ (IFN$\gamma$ ), a cytokine which was shown to promote induction of RNS in brain [36]. The highly abundant mitochondria in brain cells are a major site of generation and action of ROS/RNS. Specific forms of ROS and RNS include hydrogen peroxide $\left(\mathrm{H}_{2} \mathrm{O}_{2}\right)$, superoxide $\left(\mathrm{O}_{2}^{-}\right)$, nitric oxide $(\mathrm{NO})$, peroxynitrite $\left(\mathrm{ONOO}^{-}\right)$. Oxidative stress occurs in cells and tissues because of overproduction of superoxide $\left(\mathrm{O}_{2}{ }^{-}\right)$and its secondary oxidants that are formed from several sources, including NADPH oxidase, xanthine oxidase, and the mitochondrial electron transport chain [37].

$\mathrm{NO}$ reacts with $\mathrm{O}_{2}^{-}$and generates $\mathrm{ONOO}^{-}$, which is capable of initiating further protein oxidation and nitration. The addition of NO to thiol groups on proteins, S-nitrosation (also referred to as S-nitrosylation), has also been reported in neurodegenerative diseases [38]. GSNO, a modulator of cellular redox, is a physiological metabolite produced by the reaction of nitric oxide (NO) with glutathione (GSH). It is an 


\section{Indo Global Journal of Pharmaceutical Sciences, 2018; 8(3): 108-114}

efficient nitrosylating agent, and the mechanism of nitrosylation modulates protein functioning in health and disease. Pharmacologically, GSNO has been shown to protect the central nervous system (CNS) against excitotoxicity, inflammation, and reactive oxygen species (ROS) in a variety of injury conditions mainly through the down regulation of the expression of NF- $\mathrm{KB}$, adhesion molecules, cytokines and inducible NOS (iNOS) and neuroprotective effects via reducing the neuronal apoptotic cell death and inhibiting the activity of caspase-3 [39]. MPTP toxicity could not be induced in nNOS knocked out mice and thus 7-Nitroindazole (7-NI), a specific NOS inhibitor, also dose dependently inhibited the MPTP induced dopamine depletionand prevented the increase in peroxynitrite induced 3-NT formation in mice [2].

However the production of the NO exerts the beneficial effects initially when produces in the lower concentrations. $\mathrm{Ca}^{2+}$ influx after NMDA-receptor activation results in increased NOS activity- hence increased NO production and loss of membrane potential in mitochondria. Loss of membrane potential decreases $\mathrm{Ca}^{2+}$ uptake by mitochondria, which decreases mitochondrial NO production, salvages mitochondrial function, and protects neurons from NMDA toxicity. However, in mature hippocampal neurons, NO production after NMDA activation does not depend on mitochondrial membrane potential because the active NOS is localized to the cytosol rather than the mitochondria. Thus, the feedback effect of NO on mtNOS is not relevant and cannot protect the neurons from $\mathrm{Ca}^{2+}[32]$.

NO produced in mitochondria and is an important modulator of $\mathrm{O}_{2}{ }^{-}$production, as the ETC contains several NO reactiveredox metal centers. At physiological concentrations, NO modulates mitochondrial oxygen consumption by inhibiting cytochrome $c$ oxidase in a reversible process. Also, $\mathrm{NO}^{\circ}$ undergoes radical-radical reaction with $\mathrm{O}_{2}{ }^{-}$at near diffusion-limited rates forming peroxynitrite $\left(\mathrm{ONOO}_{2}{ }^{-}\right)$, an oxidant capable of irreversible nitration of proteins, inactivation of enzymes, DNA damage, and disruption of mitochondrial integrity [40]. Nitrite-dependent S-nitrosation of mitochondrial complex I may play a role in this phenomenon, the mechanism by which nitrite mediates cytoprotection. Complex I is particularly susceptible to ischemic damage and is a major target for $\mathrm{NO}$ and its metabolites. Several studies demonstrate that S-nitrosation of critical thiols on the complex can inhibit the enzyme, resulting in cytoprotection Inhibition of complex I activity appears to be cytoprotective due to its inhibitory effect on ROS generation, particularly at the time of reperfusion when rapid entrance of oxygen into the electron chain results in a burst of ROS formation [41]. The ATP synthase plays a vital role in cellular energetics as it is the predominant site of ATP formation in the cell. increased ATP hydrolysis by the ATP synthase during ischemia contributes to the depletion of the ATP pool. Thus, downregulation of the enzymatic activity of the ATP synthase may be beneficial through the preservation of cellular ATP. ATP synthase alpha subunit shows increased nitration after IR. GSNO-dependent preconditioning induced a concentration dependent S-nitrosation of the F1-ATP synthase alpha subunit, which inhibited the activity of the complex. The NO or nitrite dependent preservation of ATP in the ischemic tissue may contribute to the cytoprotective mechanism of IPC [42]. Snitrosation of glyceraldehyde-3-phosphate dehydrogenase (GAPDH) inhibits its dehydrogenase activity and induces an acyl phosphatase activity in the enzyme, resulting in the uncoupling of glycolytic flux from ATP synthesis. Nitrosated GAPDH can also translocate to the nucleus, enabling it to degrade selected target proteins and affect apoptosis [43].

Mitochondria served as critical for the apoptotic process and integrates death signals engaged by proteins in the Bcl-2 family and releases pro-apoptotic molecules residing in the mitochondrial intermembrane space to activate caspases leading to internucleosomal cleavage of DNA. Apoptotic stimuli produce conformational changes and oligomerization of pro-apoptotic proteins (Bax, Bak, etc.) and they cause destabilizing of lipid bilayer, creating pores or interacting with channels which results into the release of cytochrome $c$ into the cytoplasm, which (in the presence of dATP) induces the formation of the Apaf1-containing macromolecular complex called the apoptosome. Released cytochrome $c$ then triggers the assembly of the cytoplasmic apoptosome a protein complex of apoptotic protease activating factor 1 (Apaf1), cytochrome $c$, and procaspase-9 and activates caspase-3 [34]. $\mathrm{NO}$ abrogates cytochrome $\mathrm{c}$ release is through its nitrosylation of the heme in cytochrome $c$, resulting in the inhibition of its peroxidase activity and subsequent oxidation of $\mathrm{CL}$. Therefore, nitrosylation of CL-bound cytochrome $c$ represents a mechanism through which $\mathrm{NO}$ prevents the initiation of the intrinsic apoptotic pathway. NO-mediated gradual wake-up of the electron transport chain could indirectly prevent mPTP formation by attenuating mitochondrial $\mathrm{Ca}^{2+}$ overload and ROS overproduction [44]. Bcl-2 is a key apoptosis-regulatory protein of the mitochondrial death pathway. NO, through its ability to $S$-nitrosylate Bcl-2, may interfere with the ubiquitination process and inhibit proteasomal degradation of the protein [45].

NO at low doses may have antiapoptotic characteristics by blocking caspase activity via $S$-nitrotyrosylation [46]. Most of the caspases contain a single cysteine at the catalytic site, which is susceptible to redox modification and can be 


\section{Indo Global Journal of Pharmaceutical Sciences, 2018; 8(3): 108-114}

effectively modified by $S$-nitrosylation in the presence of $\mathrm{NO}$ with the subsequent inhibition of enzyme activity. NO reportedly inhibits the enzymatic activity of caspase- 3 and -8 via $S$-nitrosylation of active-site cysteine residues and suppresses apoptosis [35].

In the light of above findings it is suggested that the $\mathrm{NO}$ is implicated in the pathogenesis of PD.

\section{REFERENCES}

1. Gao HM, Kotzbauer PT, Uryu K, Leight S, Trojanowski JQ, Lee VM. Neuroinflammation and oxidation/nitration of $\alpha$-synuclein linked to dopaminergic neurodegeneration. The Journal of Neuroscience 2008;28(30):7687-98.

2. Shukla R. Nitric oxide in neurodegeneration. Annals of Neurosciences 2007;14(1):13-20.

3. Chinta SJ, Ganesan A, Reis-Rodrigues P, Lithgow GJ, Andersen JK. Anti-inflammatory role of the isoflavone diadzein in lipopolysaccharide-stimulated microglia: implications for Parkinson's disease. Neurotoxicity Research 2013;23(2):145-53.

4. Zhang F, Zhou H, Wilson BC, Hong J-S, Gao H-M. Fluoxetine protects neurons against microglial activation-mediated neurotoxicity. Parkinsonism Relat. Disord., 2012; 18(01): S213S217.

5. Pal R, Miranda M, Narayan M. Nitrosative stress-induced Parkinsonian Lewy-like aggregates prevented through polyphenolic phytochemical analog intervention. Biochem. Biophys. Res. Commun., 2011; 404(1): 324-329.

6. Zhou C, Huang Y, Przedborski S. Oxidative stress in parkinson's disease: a mechanism of pathogenic and therapeutic significance. Ann. N.Y. Acad. Sci., 2008; 1147: 93-104.

7. Ischiropoulos H, Beckman JS. Oxidative stress and nitration in neurodegeneration: cause, effect, or association? The Journal of Clinical Investigation 2003;111(2):163-9.

8. Zhang W, Phillips K, Wielgus AR, Liu J, Albertini A, Zucca FA, Faust R, Qian SY, Miller DS, Chignell CF, Wilson B. Neuromelanin activates microglia and induces degeneration of dopaminergic neurons: implications for progression of Parkinson's disease. Neurotoxicity Research 2011;19(1):63-72.

9. Stone DK, Reynolds AD, Mosley RL, Gendelman HE. Innate and adaptive immunity for the pathobiology of Parkinson's disease. Antioxidants \& Redox Signaling 2009;11(9):2151-66.

10. Englaro W, Bahadoran P, Bertolotto C, Busca R, Derijard B, Livolsi A, Peyron J-F, Ortonne J-P, Ballotti R. Tumor necrosis factor alpha-mediated inhibition of melanogenesis is dependent on nuclear factor kappa B activation. Oncogene, 1999; 18: 15531559 .

11. https://www.qiagen.com/us/shop/genes-and-pathways/pathwaydetails/?pwid=437 Accessed on 20.03.2018

12. https://www.thermofisher.com/in/en/home/lifescience/antibodies/antibodies-learning-center/antibodiesresource-library/cell-signaling-pathways/tnf-signalingpathway.html Accessed on 20.03.2018

13. Munhoz CD, Garcia-Bueno B, Madrigal JL, Lepsch LB, Scavone C, Leza JC. Stress-induced neuroinflammation: mechanisms and new pharmacological targets. Brazilian Journal of Medical and Biological Research 2008;41(12):1037-46.

14. Whitton PS. Inflammation as a causative factor in the aetiology of Parkinson's disease. British Journal of Pharmacology 2007;150(8):963-76.

15. Drechsel DA, Patel M. Role of reactive oxygen species in the neurotoxicity of environmental agents implicated in Parkinson's disease. Free Radical Biology and Medicine 2008;44(11):187386.

16. Fang J, Nakamura T, Cho DH, Gu Z, Lipton SA. S-nitrosylation of peroxiredoxin 2 promotes oxidative stress-induced neuronal cell death in Parkinson's disease. Proceedings of the National Academy of Sciences 2007;104(47):18742-7.

17. Tsang AH, Lee YI, Ko HS, Savitt JM, Pletnikova O, Troncoso JC, Dawson VL, Dawson TM, Chung KK. S-nitrosylation of XIAP compromises neuronal survival in Parkinson's disease. Proceedings of the National Academy of Sciences 2009;106(12):4900-5.

18. Schulz-Schaeffer WJ. The synaptic pathology of $\alpha$-synuclein aggregation in dementia with Lewy bodies, Parkinson's disease and Parkinson's disease dementia. Acta Neuropathol., 2010; 120 (2): 131-143.

19. Danzer KM, McLean PJ. Drug targets from genetics: alphasynuclein. CNS \& Neurological Disorders-Drug Targets 2011;10(6):712-23.

20. Conway KA, Harper JD, PT, Lansbury J. Fibrils formed in vitro from a-synuclein and two mutant forms linked to Parkinson's disease are typical amyloid. Biochemistry. 2000; 39: 25522563.

21. Paik S.R., Lee D., Cho H.J., Lee E.N., Chang C.S. Oxidized glutathione stimulated the amyloid formation of $\alpha$-synuclein. FEBS Lett. 2003;537:63-67.

22. Marques O, Outeiro TF. Alpha-synuclein: from secretion to dysfunction and death. Cell Death \& Disease 2012;3(7):e350.

23. Venda LL, Cragg SJ, Buchman VL, Wade-Martins R. $\alpha$ Synuclein and dopamine at the crossroads of Parkinson's disease. Trends in Neurosciences 2010;33(12):559-68.

24. Danielson SR, Andersen JK. Oxidative and nitrative protein modifications in Parkinson's disease. Free Radical Biology and Medicine 2008;44(10):1787-94.

25. Saha RN, Pahan K. Regulation of inducible nitric oxide synthase gene in glial cells. Antioxidants \& Redox Signaling 2006;8(56):929-47.

26. Glass CK, Saijo K, Winner B, Marchetto MC, Gage FH. Mechanisms underlying inflammation in neurodegeneration. Cell 2010;140(6):918-34.

27. Forstermann U, Sessa WC. Nitric oxide synthases: regulation and function. European Heart Journal 2012;33(7):829-37.

28. Wang M, Roberts DL, Paschke R, Shea TM, Masters BS, Kim JJ. Three-dimensional structure of NADPH-cytochrome P450 reductase: prototype for FMN-and FAD-containing enzymes. Proceedings of the National Academy of Sciences 1997;94(16):8411-6.

29. Harms AS, Barnum CJ, Ruhn KA, et al. Delayed DominantNegative TNF Gene Therapy Halts Progressive Loss of Nigral Dopaminergic Neurons in a Rat Model of Parkinson's Disease. Molecular Therapy. 2011; 19(1):46-52. doi:10.1038/mt.2010.217.

30. Chinta SJ, Andersen JK. Redox imbalance in Parkinson's disease. Biochimica et Biophysica Acta (BBA)-General Subjects 2008;1780(11):1362-7.

31. Hyun DH, Lee M, Hattori N, Kubo SI, Mizuno Y, Halliwell B, Jenner P. Effect of wild-type or mutant Parkin on oxidative damage, nitric oxide, antioxidant defenses, and the proteasome. Journal of Biological Chemistry 2002;277(32):28572-7.

32. Knott AB, Bossy-Wetzel E. Nitric oxide in health and disease of the nervous system. Antioxidants \& Redox Signaling 2009;11(3):541-53.

33. Cali T, Ottolini D, Brini M. Mitochondrial $\mathrm{Ca}^{2+}$ and neurodegeneration. Cell Calcium 2012;52(1):73-85.

34. Martin LJ. Biology of mitochondria in neurodegenerative diseases. Progress in Molecular Biology and Translational 
Indo Global Journal of Pharmaceutical Sciences, 2018; 8(3): 108-114

Science, 2012; 107: 355-415. DOI: 10.1016/B978-0-12-3858832.00005-9

35. Shahani N, Sawa A. Protein S-nitrosylation: role for nitric oxide signaling in neuronal death. Biochimica et Biophysica Acta (BBA)-General Subjects 2012;1820(6):736-42.

36. Pimentel C, Batista-Nascimento L, Rodrigues-Pousada C, Menezes RA. Oxidative Stress in Alzheimer's and Parkinson's Diseases: Insights from the Yeast Saccharomyces cerevisiae. Oxidative Medicine and Cellular Longevity. 2012;2012:132146. doi:10.1155/2012/132146.

37. Zweier JL, Chen CA, Druhan LJ. S-glutathionylation reshapes our understanding of endothelial nitric oxide synthase uncoupling and nitric oxide/reactive oxygen species-mediated signaling. Antioxidants \& redox signaling 2011;14(10):1769-75.

38. Lee J, Giordano S, Zhang J. Autophagy, mitochondria and oxidative stress: cross-talk and redox signaling. Biochemical Journal, 2012; 441(2): 523-540.

39. Khan M, Sakakima H, Dhammu TS, et al. S-Nitrosoglutathione reduces oxidative injury and promotes mechanisms of neurorepair following traumatic brain injury in rats. Journal of Neuroinflammation. 2011;8:78. doi:10.1186/1742-2094-8-78.

40. Fukai T, Ushio-Fukai M. Superoxide Dismutases: Role in Redox Signaling, Vascular Function, and Diseases. Antioxidants \& Redox Signaling. 2011;15(6):1583-1606. doi:10.1089/ars.2011.3999.

41. Shiva S. Mitochondria as metabolizers and targets of nitrite. Nitric Oxide. 2010;22(2):64-74.

42. Venkatesh S, Ramachandran A, Zachariah A, Oommen A. Mitochondrial ATP synthase inhibition and nitric oxide are involved in muscle weakness that occurs in acute exposure of rats to monocrotophos. Toxicology Mechanisms and Methods 2009;19(3):239-45.

43. Santos RM, Lourenço CF, Ledo A, Barbosa RM, Laranjinha J. Nitric Oxide Inactivation Mechanisms in the Brain: Role in Bioenergetics and Neurodegeneration," International Journal of Cell Biology, 2012;2012:1-13.

44. Murillo D, Kamga C, Mo L, Shiva S. Nitrite as a mediator of ischemic preconditioning and cytoprotection. Nitric Oxide 2011;25(2):70-80.

45. Iyer AK, Azad N, Wang L, Rojanasakul Y. Role of Snitrosylation in apoptosis resistance and carcinogenesis. Nitric Oxide 2008;19(2):146-51.

46. Peeters-Scholte C, Koster J, Veldhuis W, van den Tweel E, Zhu C, Kops N, Blomgren K, Bar D, van Buul-Offers S, Hagberg H, Nicolay K. Neuroprotection by selective nitric oxide synthase inhibition at 24 hours after perinatal hypoxia-ischemia. Stroke 2002;33(9):2304-10.

Indo Global Journal of Pharmaceutical Sciences( ISSN 22491023 ; UGC Journal No.: 44477; CODEN- IGJPAI; NLM ID: 101610675) indexed and abstracted in EMBASE(Elsevier), UGC Journal List, National Library of Medicine (NLM) Catalog, Elsevier( EMBASE), ResearchGate, Publons, CAS (ACS), Index Copernicus, Google Scholar and many more. For further details, visit http://iglobaljournal.com 